\title{
Effect of bunch shape on its transverse mode coupling instability spectrum and threshold with high space charge
}

\author{
V. Balbekov* \\ Fermi National Accelerator Laboratory, P.O. Box 500, Batavia, Illinois 60510, USA
}

(Received 24 October 2018; published 30 April 2019)

\begin{abstract}
The transverse mode coupling instability of a bunched beam is investigated in the paper at different forms of the bunches, with space charge included. An equation of transverse motion of the bunch in the parabolic potential well of synchrotron oscillations is derived and analyzed. The bunch of constant density (flat bunch) is examined in detail to make a comparison with the square well model. It is shown that both models result in very close instability thresholds of the flat bunch. Then different form bunches are investigated in the parabolic potential well. It is shown that decrease of the bunch RMS length leads to the growth of its threshold, that is the flat bunch model gives only a minimal estimation of the threshold. The results are treated in terms of Landau damping due to a spread of the space charge tune shift.
\end{abstract}

DOI: 10.1103/PhysRevAccelBeams.22.044401

\section{INTRODUCTION}

Transverse mode coupling instability (TMCI) of the bunched beam has been observed first in the electron storage ring PETRA and explained by Kohaupt [1]. Using the two-particle model, the author has shown that the instability occurs when tunes of two head-tail modes approach each other being shifted by the bunch wakefield. The shift should be about the synchrotron tune to reach the betatron tune coalescence and the beam instability.

In proton rings, the space charge (SC) tune shift $\Delta Q_{\mathrm{sc}}$ has to be taken into account as well because it typically exceeds the synchrotron tune $Q_{s}$. This effect has been considered first by Blaskiewicz [2,3]. The main point of the papers is that the SC pushes upward the TMCI threshold, that is it improves the beam stability. However, relatively small SC tune shift was considered in these works.

Different wakes were considered in the subsequent papers [4-9] at the unlimited value of the SC tune shift. It was shown that, at increasing tune shift, the TMCI threshold goes up if the wake is negative, and goes down if it is positive (the wake sign coincides here with sign of the tune shift for a rigid bunch oscillation). The effect of a resonant wake is more complicated being dependent on its phase advance in the bunch $[8,9]$.

Another result has been represented in Ref. [10]. According to it, the stable and unstable zones alternately

\footnotetext{
*balbekov@fnal.gov

Published by the American Physical Society under the terms of the Creative Commons Attribution 4.0 International license. Further distribution of this work must maintain attribution to the author(s) and the published article's title, journal citation, and DOI.
}

replace each other when the SC tune shift increases, even at the constant wakefield. The two-particle approximation has been used in the paper like the pioneer work [1], with a model of the space charge field proposed. Further examination of the model looks to be needed.

It should be noted that most of the mentioned results were obtained in the framework of the flat (square) bunch model. It ignores dependence of the SC tune shift of particles on their longitudinal position, and related betatron tune spread. Meanwhile, it is known that the spread due to nonlinearity or chromaticity affects the transverse instability thresholds by a redistribution of the tunes [11]. The similar effect should not be excluded if the spread is caused by space charge. This problem is investigated in this paper.

In Sec. II, the physical model is introduced, and the equation of transverse oscillations of an arbitrary bunch with the wakefield and the space charge is proposed.

In Sec. III, the equation is applied to the flat bunch to compare the results with the known models and to check their identity and applicability.

In Sec. IV, the equation is applied to different bunch forms to investigate their spectra and thresholds in comparison with the flat bunch.

\section{PHYSICAL MODEL}

\section{A. General}

We will consider coherent transverse oscillations of a bunch in the rest frame, representing its displacement in a point of the longitudinal phase space as real part of the function

$$
X=Y(\theta, u) \exp \left[-i\left(Q_{0}+\xi\right) \theta-i\left(Q_{0}+\nu\right) \Omega_{0} t\right]
$$


where $\theta=z / R_{0}$ and $u=\dot{\theta}$ are the longitudinal coordinate and corresponding velocity, $R_{0}$ and $\Omega_{0}$ are the accelerator average radius and central revolution frequency, $Q_{0}$ and $\nu$ are the bare betatron tune and the addition to it caused by the collective effects, and $\xi$ is the normalized chromaticity:

$$
\xi=-\frac{d Q_{0} / d \ln (u)}{\alpha-1 / \gamma^{2}}
$$

with $\alpha$ as the momentum compaction factor. The slowly changing amplitude of the betatron oscillations $Y$ satisfies the equation [5]

$$
\begin{aligned}
\nu Y & +i Q_{s} \frac{\partial Y}{\partial \phi}+\Delta Q_{\mathrm{sc}}(Y-\bar{Y}) \\
& =2 \int_{\theta}^{\infty} q\left(\theta^{\prime}-\theta\right) \exp \left[-i \xi\left(\theta^{\prime}-\theta\right)\right] \bar{Y}\left(\theta^{\prime}\right) \rho\left(\theta^{\prime}\right) d \theta^{\prime}
\end{aligned}
$$

where $\phi$ and $Q_{s}$ are phase and tune of the synchrotron oscillations. The space charge tune shift $\Delta Q_{\mathrm{sc}}(\theta)$ does not depend on transverse coordinates because it is an average over the beam cross section [12]. The normalized wake field potential $q(\theta)$ is proportional to usual transverse wake function $W_{1}(z)$ :

$$
q(\theta)=\frac{r_{0} N_{b} R_{0} W_{1}\left(-R_{0} \theta\right)}{8 \pi \beta^{2} \gamma Q_{0}}
$$

with $r_{0}=e^{2} / m c^{2}$ as the particle electromagnetic radius, $\beta$ and $\gamma$ as its normalized velocity and energy, and $N_{b}$ as the bunch population. Besides, the notation and the normalization condition are used in Eq. (3):

$$
\begin{aligned}
\int_{-\infty}^{\infty} F(\theta, u) Y(\theta, u) d u & =\rho(\theta) \bar{Y}(\theta), \\
\int_{-\infty}^{\infty} F(\theta, u) d u & =\rho(\theta), \\
\int_{-\infty}^{\infty} \rho(\theta) d \theta & =1
\end{aligned}
$$

where $F$ is the bunch distribution function in the longitudinal phase space.

\section{B. Used simplifications}

Being interested mainly by dependence on the TMCI threshold on the SC tune shift at arbitrary bunch shape, we restrict ourselves to the case of zero chromaticity and constant wake: $\xi=0, q=q_{0}=$ const. Besides, we will consider only linear synchrotron oscillations taking $Q_{s}=1$ in the equations. Finally, we will consider a bunch of restricted length, and will take new longitudinal coordinate and velocity $x \propto \theta, v=\dot{x}$, to have the bunch location in the region $-1<x<1$. Then Eq. (3) obtains the form $\nu Y+i \frac{\partial Y}{\partial \phi}+\Delta Q_{\mathrm{sc}}(Y-\bar{Y})=2 q_{0} \int_{x}^{1} \bar{Y}\left(x^{\prime}\right) \rho\left(x^{\prime}\right) d x^{\prime}$

with the normalization conditions replacing Eq. (5)

$$
\begin{aligned}
\int F\left(x^{2}+v^{2}\right) Y(x, v) d v & =\rho(x) \bar{Y}(x), \\
\int F\left(x^{2}+v^{2}\right) d v & =\rho(x), \\
\int_{-1}^{1} \rho(x) d x & =1 .
\end{aligned}
$$

Parameter $q_{0}$ is proportional to an average value of the original wake potential $W_{1}$, if the last is a monotonous function of the coordinate (for example the resistive wall wake). The proportionality coefficient is clear from Eq. (6) itself because it should give the tune shift $\nu=q_{0}$ for the lowest (rigid) head-tail mode $Y=\bar{Y}=1$ :

$$
\begin{aligned}
\nu & =2 \int_{-1}^{1} \rho(x) d x \int_{x}^{1} q\left(x-x^{\prime}\right) \rho\left(x^{\prime}\right) d x^{\prime} \\
& \rightarrow 2 q_{0} \int_{-1}^{1} \rho(x) d x \int_{x}^{1} \rho\left(x^{\prime}\right) d x^{\prime}=q_{0} .
\end{aligned}
$$

\section{High space charge approximation}

Furthermore, it is convenient to consider only the even part of the function $Y$ which has the form $Y^{+}(\phi)=$ $[Y(\phi)+Y(-\phi)] / 2$ and satisfies the equation [9]:

$\frac{\partial}{\partial \phi}\left(\frac{\partial Y^{+}}{\hat{\nu} \partial \phi}\right)+\hat{\nu} Y^{+}=\Delta Q_{\mathrm{sc}} \bar{Y}+2 q_{0} \int_{x}^{1} \bar{Y}\left(x^{\prime}\right) \rho\left(x^{\prime}\right) d x^{\prime}$

where $\hat{\nu}(x)=\nu+\Delta Q_{\mathrm{sc}}(x)$. Multiplying this equation by the function $F$, integrating on $v$, using Eq. (7), and taking into account the relations

$$
\frac{\partial}{\partial \phi}=v \frac{\partial}{\partial x}-x \frac{\partial}{\partial v}, \quad \frac{\partial F}{\partial \phi}=0
$$

we obtain

$$
\begin{aligned}
& \frac{d}{d x}\left(\frac{d}{\hat{\nu} d x} \int Y^{+} F v^{2} d v\right)+\frac{d}{d x}\left(\frac{x \rho \bar{Y}}{\hat{\nu}}\right)+\nu \rho \bar{Y} \\
& \quad=2 q_{0} \rho(x) \int_{x}^{1} \bar{Y}\left(x^{\prime}\right) \rho\left(x^{\prime}\right) d x^{\prime} .
\end{aligned}
$$

A similar equation has been proposed earlier in the framework of the square potential well model, where the positive phase can be identified with the longitudinal coordinate [6]. The goal of this paper is to extend this to arbitrary potential well using the high space charge approximation developed in papers $[4,12]$. The main idea 
is that at rather large $\Delta Q_{\mathrm{sc}}$, the function $Y^{+}$depends on coordinate $x$ essentially stronger than on velocity $v$. In accordance with this, one can put $Y^{+} \simeq \bar{Y}$ in the left-hand part of Eq. (10). It results in the equation for the bunch dipole momentum $D(x)=\rho(x) \bar{Y}(x)$ :

$\frac{d}{d x}\left(\frac{d\left(U^{2} D\right)}{\hat{\nu} d x}\right)+\frac{d}{d x}\left(\frac{x D}{\hat{\nu}}\right)+\nu D=2 q_{0} \rho(x) \int_{x}^{1} D\left(x^{\prime}\right) d x^{\prime}$

where $U(x)$ is the RMS value of normalized momentum of the particles at the longitudinal coordinate $x$ :

$$
U^{2}(x)=\frac{1}{\rho(x)} \int_{-1}^{1} F\left(x^{2}+v^{2}\right) v^{2} d v
$$

It is easy to check that

$$
\frac{d\left(\rho U^{2}\right)}{d x}=-x \rho(x)
$$

A usage of this relation in Eq. (11) would allow us to get a final form of this integral-differential equation. However, it is more convenient for the numerical solution to represent it as the system of three first order equations

$$
\begin{aligned}
\frac{d D}{d x} & =\frac{\hat{\nu}(x) D_{1}(x)}{U^{2}(x)} \\
\frac{d D_{1}}{d x} & =\left[\frac{d}{d x}\left(\frac{U^{2} \rho^{\prime}(x)}{\hat{\nu} \rho}\right)-\nu\right] D+2 q_{0} \rho D_{2}, \\
\frac{d D_{2}}{d x} & =-D(x) .
\end{aligned}
$$

The incoming functions satisfy the relations $\rho(x)=0$ and $U^{2}(x)=0$ at $x= \pm 1$. Because the bunch dipole momentum $D(x)$ and its derivative are expected to be constrained values on any point of the space, the following boundary conditions must be satisfied:

$$
D_{1}( \pm 1)=0, \quad D_{2}(1)=0 .
$$

The problem has a countless number of eigenfunctions and corresponding eigentunes. The real eigentunes can be found by means of the following steps as it has been proposed and applied in Refs. [6,9]:

(1) At given value of the SC tune shift, and with a trial value of the wake $q_{0}$, series of Eq. (14) should be resolved step by step by moving from the bunch end $x=1$ to its beginning $x=-1$, with initial conditions $D(1)=1$, $D_{1}(1)=0, D_{2}(1)=0$.

(2) The tune $\nu$ should be varied to find the value providing fulfillment of the boundary condition $D_{1}(-1)=0$.
(3) Steps 1-2 should be repeated with different $q_{0}$ so many times to obtain several curves $\nu_{i}\left(q_{0}\right)$ describing the dependence of eigenvalues on the wake strength at given $\Delta Q_{\mathrm{sc}}$.

(4) The instability threshold of each mode should be determined as the curve turning point.

The operations should be repeated with all desirable tune shifts. Note that the condition $\hat{\nu}(x) \neq 0$ is assumed to be fulfilled at any step of the solving. This important point will be discussed in detail in Sec. IV.

\section{FLAT BUNCH}

The TMCI threshold of a flat bunch $(\rho=$ const $)$ was considered earlier using different approximations, including the expansion technique and/or the square well model $[2,4,7,9]$. However, the case of linear synchrotron oscillations has been analyzed only at a modest value of the SC tune shift. Equation (14) allows us to consider the problem more widely.

The involved functions are at $|x|<1$

$$
\begin{aligned}
\rho(x) & =\frac{1}{2}, \\
U^{2}(x) & =\frac{1-x^{2}}{2}, \\
\hat{\nu} & =\nu+\Delta Q_{\mathrm{sc}}=\text { const. }
\end{aligned}
$$

Using the notations

$$
\mathcal{P}=\hat{\nu} \nu=\hat{\nu}\left(\hat{\nu}-\Delta Q_{\mathrm{sc}}\right), \quad \mathcal{Q}=q_{0} \hat{\nu}
$$

one can reduce Eq. (11) to the form which does not include the SC tune shift explicitly:

$$
\frac{d}{d x}\left(U^{2} \frac{d D}{d x}\right)+\mathcal{P} D=\mathcal{Q} \int_{x}^{1} D\left(x^{\prime}\right) d x^{\prime}
$$

A similar equation has been obtained in Ref. [6] on the base of the square well model. With an equalization of the bunch length, the only remaining difference would be the factor $4 / \pi^{2}$ instead of $U^{2}(x)=\left(1-x^{2}\right) / 2$ in Eq. (18).

Solution of the equation by the above-described method provides an infinite set of the eigentunes $\mathcal{P}_{n}$ at each $\mathcal{Q}$. They form the lines in the $(\mathcal{Q}-\mathcal{P})$ plane some of which are shown in Fig. 1. Note that, at $\mathcal{Q}=0$, Eq. (18) is Legendre equation with eigennumbers $\mathcal{P}_{m}=0,1,3,6, \ldots m(m+1) / 2, \ldots$. The lines in Fig. 1 cross the vertical axis $\mathcal{Q}$ just in these points so that the numbers $m$ can be treated as the indexes of the lower multipole in the solution. At $\mathcal{Q}>0$, some lines merge in pairs which will be marked further by symbols $M_{0,1}, M_{2,3}$ etc. For example, the red lines link up at $\mathcal{Q}=0.468$ producing the $M_{0,1}$ coupled mode. Actually, the lines extend to the region $\mathcal{Q}>0.468$ as the complex-conjugate pair, that 


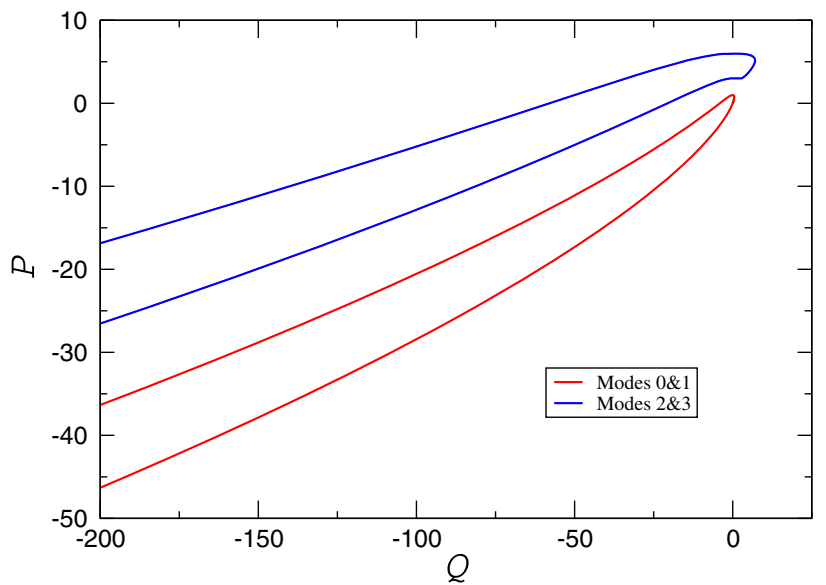

FIG. 1. Some eigentunes of Eq. (18) against the referred wake strength. The red line $M_{0,1}$ illustrates coalescence of the lowest head-tail pair, the blue line $M_{2,3}$ does this for the next pair.

is $\mathcal{Q}_{0,1}=0.468$ is the critical point of the $M_{0,1}$ mode. Higher modes have higher critical points: for example $\mathcal{Q}_{2,3}=7.07$ (blue lines in Fig. 1), $\mathcal{Q}_{4,5}=28.6$, etc.

According to Eq. (17), each curve of the $(\mathcal{Q}-\mathcal{P})$ plane is mapped to 2 curves in the $\left(q_{0}-\hat{\nu}\right)$ plane, dependent on $\Delta Q_{\mathrm{sc}}$ :

$$
\hat{\nu}=\frac{\Delta Q_{\mathrm{sc}}}{2} \pm \sqrt{\frac{\Delta Q_{\mathrm{sc}}^{2}}{4}+\mathcal{P}}, \quad q_{0}=\frac{\mathcal{Q}}{\hat{\nu}}
$$

(see Fig. 2). Each obtained point is the eigentune of some head-tail mode with space charge and wakefield. There are left and right turning points in the curves where two real head-tail eigentunes join together. It means an appearance of the complex conjugated eigentunes that is the threshold of corresponding TMCI mode.

For example, at $\Delta Q_{\mathrm{sc}}=0, M_{0,1}$ mode has the turning points $q_{0} / Q_{s}=\mp 0.57$ with corresponding values of $\nu / Q_{s}=\mp 0.75$. They are the TMCI thresholds of negative or positive wake without space charge (the black line in the upper panel of Fig. 2). The curves stretch to the left-down direction when the SC tune shift increases, resulting in a movement of the turning points. Change of the TMCI threshold in the process depends on the wake sign: threshold of the positive wake decreases going to 0 at $\Delta Q_{\text {sc }} \rightarrow \infty$ whereas the threshold of the negative wake $\left|q_{0}\right|=-q_{0}$ tends to infinity in the case. Similar behavior of the $M_{2,3}$ mode is shown in the lower panel of Fig. 2.

The more complicated case of the negative wake is additionally illustrated by Fig. 3 where the mentioned modes are shown together. When the SC tune shift increases, both turning points move to the left doing this with different velocity. The turning point of the lower mode $M_{0,1}$ moves especially quickly, particularly because of fast growth of distance between initial points of the loop in the vertical axis, which always exceeds $\Delta Q_{\mathrm{sc}}$. Other modes
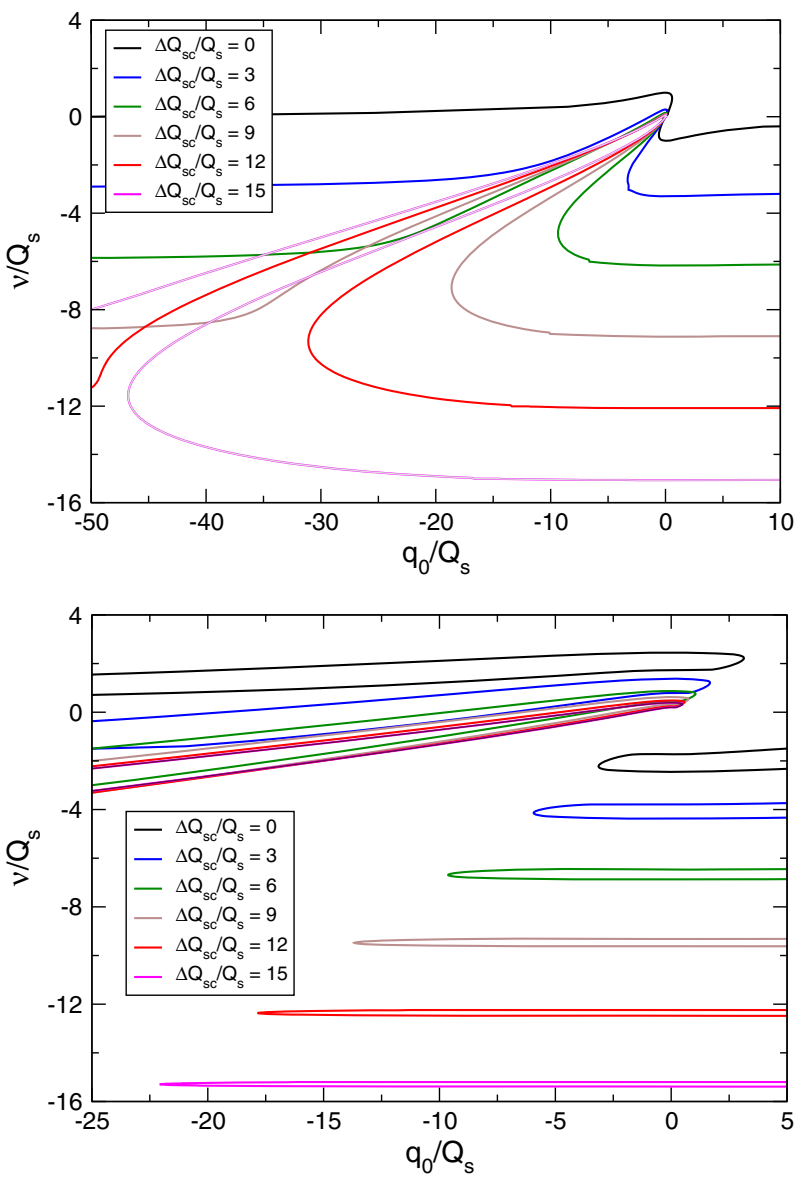

FIG. 2. Several eigentunes of the bunch against the wake strength at different value of the space charge tune shift. The lower bunch modes $M_{0,1}$ generated by the red line of Fig. 1 are plotted in the top panel, and the modes $M_{2,3}$ are plotted in the bottom panel (blue line in Fig. 1).

grow slower. As a result, the mode $M_{0,1}$ is the most unstable only at $\Delta Q_{\mathrm{sc}} / Q_{s}<6$, otherwise the higher mode $M_{2,3}$ intercepts the threshold. Still higher modes of the flat bunch have the higher thresholds.

Dependence of the TMCI threshold on the SC tune shift is shown in detail in Fig. 4, where the obtained thresholds of the $M_{0,1}$ and $M_{2,3}$ modes are plotted against the shift by the solid lines. Similar curves for the square well model have been taken in Ref. [6] and are plotted by the dashed lines. The same results could be obtained also with the help of Eq. (18) by the substitution $U^{2}=4 / \pi^{2}$. It does not affect the bunch shape but changes characteristics of synchrotron oscillations, which looks like a secondary factor in the case.

\section{NONFLAT BUNCHES}

The flat bunch model ignores the fact that the SC tune shift of a particle depends on its longitudinal coordinate which circumstance creates an additional betatron tune spread. It is known that the spread due to chromaticity or nonlinearity of betatron oscillations leads to an appearance 


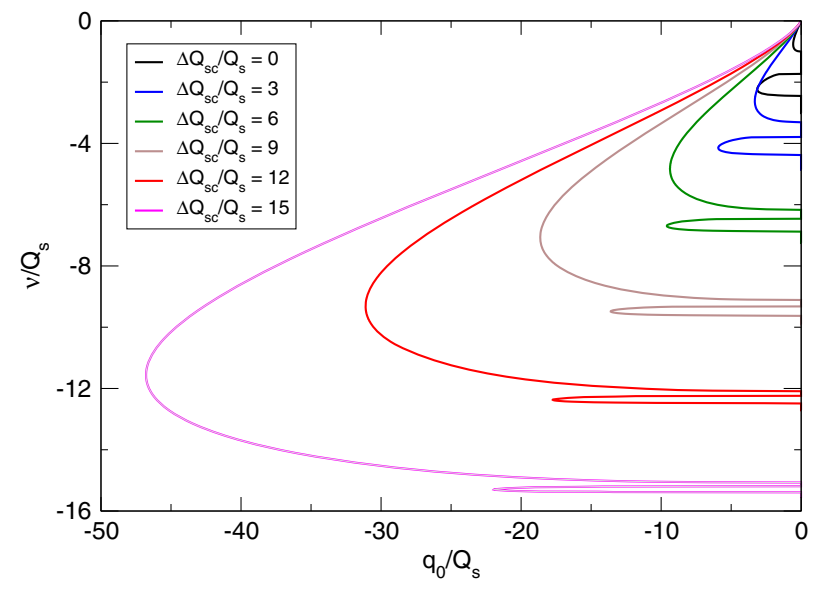

FIG. 3. Tunes of the modes $M_{0,1}$ and $M_{2,3}$ against the wake strength for the flat bunch with negative wake. The mode $M_{0,1}$ has the lowest threshold at $\Delta Q_{\mathrm{sc}} / Q_{s}<6$, otherwise the threshold of the $M_{0,1}$ mode is less. Other modes are more stable in any case.

or an increase of the transverse instability threshold. Such an effect in accelerators is known as Landau damping which is treated as not dissipative damping but simply the absence of instability due to redistribution of the particles [11]. The "redistribution" means actually an ousting of the coherent betatron frequency from the region where the particle betatron frequencies are located. As a result, the coherent frequency acquires a position outside the region, if the frequency distribution of particles is sufficiently compact, or obtains it rather far from the center $(3-5 \sigma)$ for the distributions with an infinite tail, like Gaussian. It follows from the threshold diagrams which have different asymptotic forms in the cases of the "compact" and the "tail" distributions [11].

The problem is investigated in this section with regard to the influence of the SC tune spread on the TMCI threshold. We will consider only the compact bunches with a clear boundary. Distribution of the SC tune shifts is also clearly confined in the case. Any coherent bunch oscillations are impossible in this area because their energy would transform at once in an incoherent form that is in the beam heating. Therefore an essential change of the TMCI tune and threshold has to be expected in comparison with the flat bunch.

It follows from Fig. 3 that the lowest head-tail coherent tunes of the flat bunch join together at $\nu>-\Delta Q_{\mathrm{sc}}$, that is at $|\nu|<\Delta Q_{\mathrm{sc}}$, if $\Delta Q_{\mathrm{sc}} / Q_{s}>3$. For a nonflat bunch, such a coupling would occur within the incoherent tune spread area. Indeed, the particle betatron tunes $Q_{\beta}$ are located in the area $Q_{0}-\Delta Q_{\max }<Q_{\beta}<Q_{0}$ where $\Delta Q_{\max }=\Delta Q_{\mathrm{sc}}(0)$ is the SC tune shift in the bunch center. In the used notation, it means that $-\Delta Q_{\max }<\nu_{\beta}<0$. As mentioned above, the coalescence cannot happen in this region, and a change of the tunes has to be expected. It means also a change of the wakefield required for the coalescence, that is to a change of the TMCI threshold.

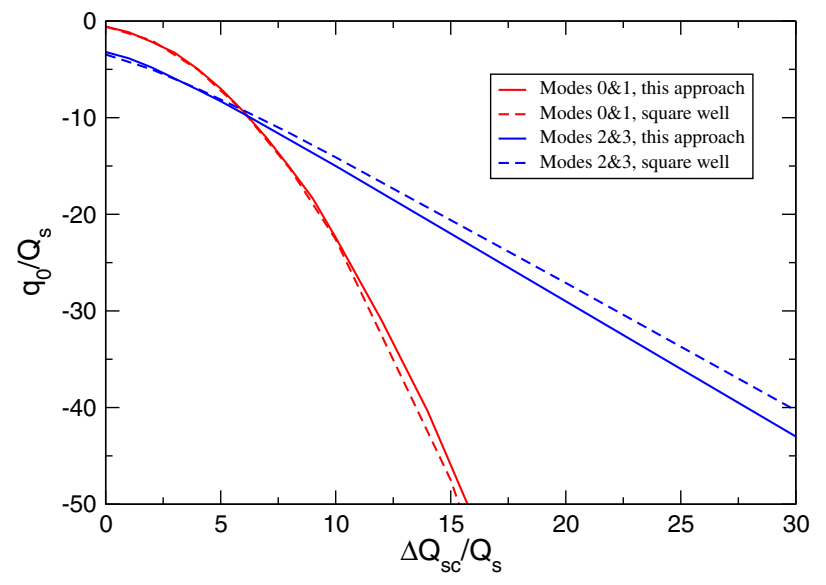

FIG. 4. TMCI thresholds of the flat bunch. Solid lines are obtained in this paper, dashed lines - with the square well model [6]. Red and blue lines represent the modes $M_{0,1}$ and $M_{2,3}$. It is seen that $M_{0,1}$ is the most unstable mode only at $\Delta Q_{\mathrm{sc}} / Q_{s}<6$, otherwise $M_{0,1}$ mode is more unstable.

Formally, the SC tune spread can lead to an appearance of a singularity in Eq. (8) and further, if $\nu$ is a real number satisfying the condition $-\Delta Q_{\max }<\nu<0$. This circumstance does not discard the method because the tune $\nu$ appears in the equations as a parameter of the Laplace transform having a positive imaginary part. However, its extension to the real axis in subsequent transformations is allowable only by the analytical continuation of appearing functions. It is seen now that the continuation is possible either at $\operatorname{Re} \nu<-\Delta Q_{\max }$ or at $\operatorname{Re} \nu>0$. The second inequality directs us to the area where a positive wake can provide the tune coalescence and the instability. In particular, it means that the TMCI threshold of positive wake cannot be very sensitive to the SC tune spread, that is to the bunch shape. Therefore only the case of negative wakes is analyzed below.

We will consider the bunch distribution functions with different parameters $\alpha$ :

$$
F=\frac{2 \alpha+1}{2 \pi}\left(1-A^{2}\right)^{\alpha-1 / 2} \times \begin{cases}1 & \text { at } A<1 \\ 0 & \text { at } A \geq 1\end{cases}
$$

where $A=\sqrt{x^{2}+v^{2}}$ is amplitude of synchrotron oscillations. Then, using Eq. (7a) and Eq. (12), we obtain

$$
\rho(x)=C_{\alpha}\left(1-x^{2}\right)^{\alpha}, \quad U^{2}(x)=\frac{1-x^{2}}{2(\alpha+1)}
$$

with the normalizing coefficient

$$
C_{\alpha}=\frac{2 \alpha+1}{2 \pi} \int_{-1}^{1}\left(1-t^{2}\right)^{\alpha-1 / 2} d t .
$$

The series of Eq. (14) with the boundary conditions given by Eq. (15) has been resolved by the method described at 
the end of Sec. II. The results are represented in Fig. 5 for the cases: $\alpha=0, C_{0}=1 / 2$ (flat bunch); $\alpha=1 / 2$, $C_{1 / 2}=2 / \pi$ (waterbag model); and $\alpha=1, C_{1}=3 / 4$ (parabolic bunch). The eigentunes are plotted in the area $-2<\nu+\Delta Q_{\max }<0$ for the negative wake.
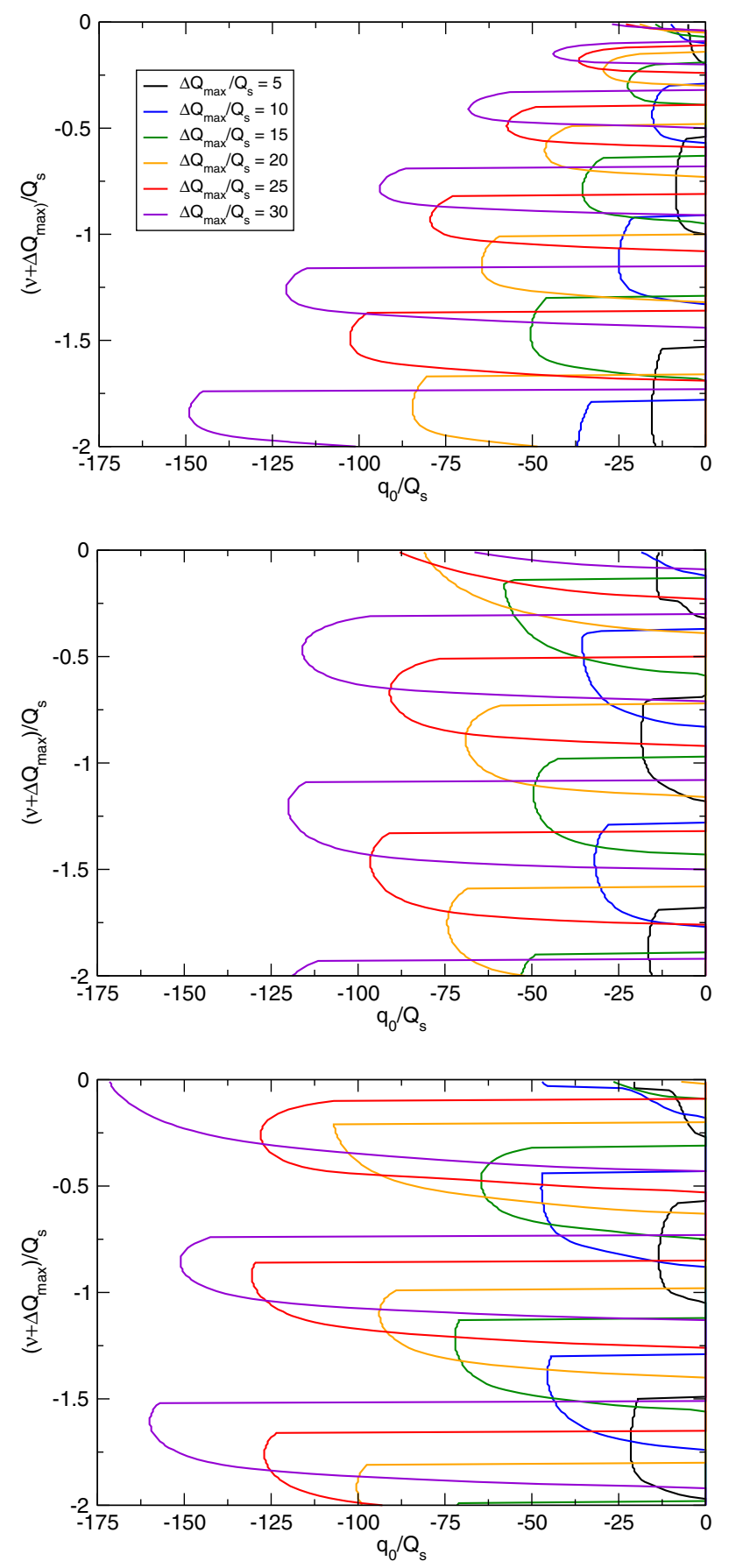

FIG. 5. The eigentunes of different bunches $\hat{\nu}_{m}=\nu_{m}+\Delta Q_{\max }$ against the wake strength $q_{0}$ at different $\Delta Q_{\max }$. The represented area $-2<\hat{\nu}_{m}<0$ is located below the particle tunes: $\hat{\nu}_{m}<\nu_{\beta}$. The top panel: flat bunch $\alpha=0$; the central panel: waterbag model $\alpha=1 / 2$; the bottom panel: parabolic bunch $\alpha=1$. The same colors are used in all the panels for the same $\Delta Q_{\max } / Q_{s}$.
TABLE I. Thresholds of negative wake $q_{0} / Q_{s}$ of the lower TMCI modes at $\Delta Q_{\max } / Q_{s}=15$ for different bunch shape.

\begin{tabular}{lccc}
\hline \hline & $M_{2,3}$ & $M_{4,5}$ & $M_{6,7}$ \\
\hline Flat & -22 & -36 & -50 \\
Waterbag & -57 & -50 & -53 \\
Parabolic & -64 & -73 & -73 \\
\hline \hline
\end{tabular}

The top panel of Fig. 5 refers again to the flat bunch. The upper loops of the graph represent eigentunes of the mode $M_{2,3}$ at different tune shifts. This mode was considered also is Sec. III where its spectrum is represented in Fig. 3 by the lower loops. The results agree closely with each other. For example, both plots give a threshold of this TMCI mode $q_{0} / Q_{s}=-22$ at $\Delta Q_{\mathrm{sc}} / Q_{s}=15$. Following modes $M_{4,5}$ and $M_{6,7}$ have essentially higher in absolute value thresholds: $q_{0} / Q_{s}=-36$ and $q_{0} / Q_{s}=-50$ (the green lines in Fig. 5).

The spectra of more realistic bunches have a similar configuration but demonstrate an essentially weaker dependence of the instability threshold on the mode number. Two of them are shown in Fig. 5. The waterbag model $\alpha=1 / 2$ is considered in the central panel, and the parabolic bunch $\alpha=1$ is represented in the lower panel. Three coupled modes are shown on each of the graphs at different $\Delta Q_{\max }$. Their thresholds are distinct from each other with no more than $10 \%$ that is essentially less than in the flat model. It is seen also that dependence of the threshold on the mode number can be nonmonotonic. The same follows from Table I where the numerical values of the threshold are given at $\Delta Q_{\max } / Q_{s}=15$.

Consideration of other distributions supports these conclusions. The results are collected in Fig. 6 where the dependence of the TMCI threshold of the most unstable mode on the maximal value of the SC tune shift is plotted for the distributions with different $\alpha$. It is seen that the TMCI threshold rises when the parameter $\alpha$ increases.

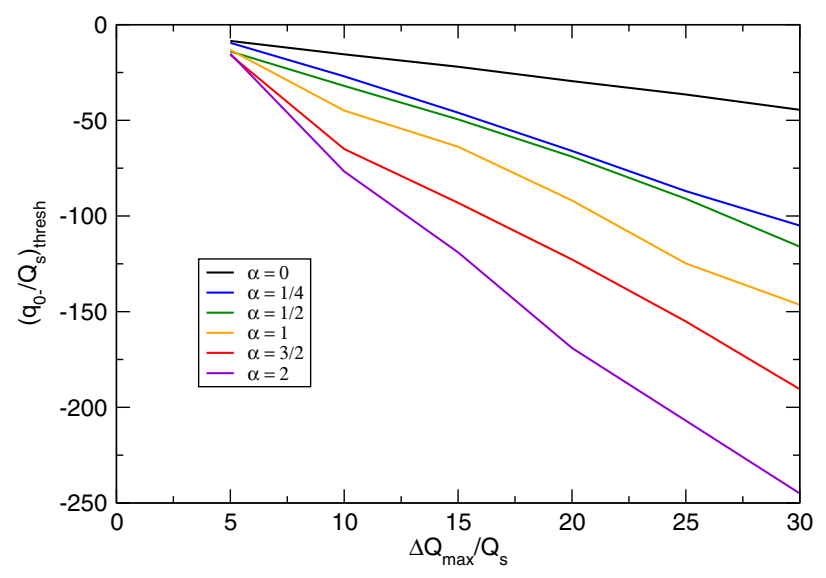

FIG. 6. TMCI thresholds of different form bunches against the SC tune shift. 
Note that the increase of $\alpha$ means the decrease of the bunch RMS length. Really, it follows from Eq. (20)

$$
\sigma^{2}=\frac{2 \alpha+1}{2 \pi} \int_{0}^{1}\left(1-A^{2}\right)^{\alpha+1 / 2} A^{3} d A \int_{-\pi}^{\pi} \cos ^{2} \phi d \phi=\frac{1}{2 \alpha+3} .
$$

It means that, at the same central density, the shorter bunch has the higher TMCI threshold, and that the flat bunch model provides only the lower estimation of the threshold.

\section{CONCLUSION}

It is shown in this paper that the TMCI thresholds of a flat bunch only slightly depends on the potential well shape being almost identical in the square well and in the parabolic one, if the same ratio of the space charge tune shift to the synchrotron tune $\Delta Q_{\mathrm{sc}} / Q_{s}$ is used. This means that the characteristic of synchrotron oscillations is the secondary factor in the case. The space charge tune shift reduces the TMCI threshold of positive wakes and increases it if the wake is negative. In the last case, the most unstable coupled modes of the flat bunch are the lowest mode $M_{0,1}$ at $\Delta Q_{\mathrm{sc}} / Q_{s}<6$, and the next mode $M_{2,3}$ at $\Delta Q_{\mathrm{sc}} / Q_{s}>6$. Corresponding coherent tunes are a little higher or lower of the particle betatron tunes, with the SC tune shift included. All other modes have a higher threshold, at any value of the SC tune shift.

A spread of the SC tune shift which is intrinsic to any real (nonflat) bunch essentially changes the bunch spectrum if the wake is negative. It forces out the coherent mode tunes from the area $-\Delta Q_{\max }<\nu<0$ where the particle incoherent tunes are located. As a result, the mode $M_{0,1}$ is excluded from the spectrum at $\Delta Q_{\max }>Q_{s}$, and tunes of other coherent modes fall below the minimal particle tune.

In such conditions, thresholds of all the TMCI modes differ from each other to only a small extension. The thresholds increases when the RMS length of the bunch decreases at fixed total length. Therefore the flat bunch model with given $\Delta Q_{\max }$ provides only the lowest estimation of the TMCI threshold.

\section{ACKNOWLEDGMENTS}

Fermi National Accelerator Laboratory is operated by Fermi Research Alliance, LLC under Contract No. DEAC02-07CH11395 with the United States Department of Energy.

[1] R. Kohaupt, in Proceeding of the XI International Particle Accelerator Conference (JACoW, Geneva, Switzerland, 1980), p. 562; DESY Report No. M-80/19 (1980).

[2] M. Blaskiewicz, Fast head-tail instability with space charge, Phys. Rev. ST Accel. Beams 1, 044201 (1998).

[3] M. Blaskiewicz, Comparing new models of transverse instability with simulations, in Proceedings of the 3rd International Particle Accelerator Conference. New Orleans, LA, 2012 (IEEE, Piscataway, NJ, 2012).

[4] A. Burov, Head-tail modes for strong space charge, Phys. Rev. ST Accel. Beams 12, 044202 (2009); Errattum, Phys. Rev. ST Accel. Beams 12, 109901 (2009).

[5] V. Balbekov, Transverse instability of a bunched beam with space charge and wakefield, Phys. Rev. ST Accel. Beams 14, 094401 (2011).

[6] V. Balbekov, Threshold of transverse mode coupling instability with arbitrary space charge, Phys. Rev. Accel. Beams 20, 114401 (2017).

[7] T. Zolkin, A. Burov, and B. Pandey, TMCI and Space Charge II, arXiv:1711.11110.

[8] A. Burov and T. Zolkin, TMCI with Resonator Wakes, Report No. FERMILAB-TM-2680-APC-CD.

[9] V. Balbekov, Transverse mode coupling instability of the bunch with oscillating wake field and space charge, Report No. FERMILAB-PUB-18-031-APC.

[10] Y.H. Chin, A. W. Chao, and M. M. Blaskiewicz, Two particle model for studying the effect of space-charge force on strong head-tail instabilities, Phys. Rev. Accel. Beams 19, 014201 (2016).

[11] Handbook of Accelerator Physics and Engineering, edited by A. Chao and M. Tigner (World Scientific, Singapore, 1998), pp. 122-125.

[12] V. Balbekov, Transverse modes of a bunched beam with space charge dominated impedance, Phys. Rev. ST Accel. Beams 12, 124402 (2009). 